\title{
Dietary Supplementary of Modified Si-jun- zi Granule Promotes Intestinal Development and Enhances Mucosal Immunity of Weaned Rex Rabbits
}

\section{Shiqi Chen}

Sichuan Agricultural University

Ning Liu

sichuan academy of grassland sciences

Dongbo Li

Sichuan Agricultural University - Chengdu Campus

Haibo Dou

Sichuan Agricultural University - Chengdu Campus

Quanjin Liu

Sichuan Agricultural University - Chengdu Campus

Hanzhong Liu

sichuan academy of grassland sciences

Ping Wang

sichuan academy of grassland sciences

\section{Zhiju Yu}

sichuan academy of grassland sciences

\section{Gang Shu}

Sichuan Agricultural University - Chengdu Campus

Juchun Lin

Sichuan Agricultural University - Chengdu Campus

Wei Zhang

Sichuan Agricultural University - Chengdu Campus

Guangneng Peng

Sichuan Agricultural University - Chengdu Campus

\section{Ling Zhao}

Sichuan Agricultural University - Chengdu Campus

Huaqiao Tang

Sichuan Agricultural University - Chengdu Campus

\section{Kai Zhang}

sichuan academy of grassland sciences 


\section{Bin Wen}

sichuan academy of grassland sciences

Hualin Fu ( $\sim$ fuhl2005@sohu.com)

Sichuan Agricultural University - Chengdu Campus

\section{Research}

Keywords: Weaned rex rabbits, Modified Si-jun-zi granule, Diarrhea, Intestinal development, Mucosal immunity

Posted Date: August 27th, 2020

DOI: https://doi.org/10.21203/rs.3.rs-64033/v1

License: (c) (i) This work is licensed under a Creative Commons Attribution 4.0 International License. Read Full License 


\section{Abstract}

Background: Early-weaned in Rex rabbits and other animals is associated with the development and immunity of intestine. This article was aimed to investigate the potential benefits of a dietary supplementation, modified Si-jun-zi granule (M-SJZG), on enhancing immunity of small intestinal mucosa and promoting intestinal development of weaned Rex rabbits.

Results: In the present study, we found that dietary supplementation with $2 \%$ modified Si-jun-zi granule decreased the diarrhea frequency and mortality due to early-weaned, and significantly increased the average daily gain; the length and relative weight of intestine; the concentration of immunoglobulin in serum and SIgA in intestinal mucus. Additionally, the activity of lipase was raised by dietary Si-jun-zi granule addition. However the activities of ALT, ALP and AST in serum were obviously decreased. Compared with the un-supplementation control group, the ratio of villi length to crypt depth and the expression of tight junction protein(zonula occludens-1, Claudin-1 and Occludin) and Glucose transporter (re-combinant Sodium/Glucose co-transporter 1 and recombinant Glucose transporter 2) in jejunum were raised. In addition, Moreover early development of intestine and the injury of intestinal mucosa induced by early-weaning also could be improved.

Conclusions. This study indicated that modified Si-jun-zi granule has protective effects on diarrhea induced by early-weaning through enhancing immunity of small intestine mucosa and promoting intestinal development.

\section{Introduction}

In general, it will take three months for natural weaning in Rex rabbits breeding. Early weaning is a commonly used practice to ensure profit maximization by shorten dams reproductive cycle(Kumar et al., 2017), after which diarrhoea and even death is often happened to induce important economic losses(Richter et al., 2017; Yarnall and Thrusfield, 2017)due to underdevelopment of digestive system and relatively low immunity(Vicario et al., 2015), especially in which food can not satisfied the requirements of nutrition(Zhu et al., 2015). The intestinal development is closely related to the permeability and nutrition absorption(Wood et al., 2015). These functions can be estimated by intestinal tight junction protein (Zo-1, Claudin-1and Occludin) level and intestinal Glucose transporter (SGLT1 and GLUT2) level.

Antibiotic was used extensively for treating and preventing diarrhoea by effectively kill microorganisms(Szajewska et al., 2016). However, it not only kills pathogens, but also causes a certain degree of damage on probiotic group that is helpful to establish the immunity barrier for intestinal mucosa(Wischmeyer et al., 2016). In addition, antibiotic residues, antibiotic resistant bacteria and antibiotic resistance genes as the important factors for common's health has attracted more and more public attention. It has become the universal goal for breeding industry to reduce the antibiotic abuse. Therefore, traditional Chinese herbal compound with some advantages, natural, low toxicity and no 
residue(Liu et al., 2015) have been widely concerned as the potential replacements to cure and prevent diarrhoea induced by early weaning.

In traditional Chinese medicine(TCM) theory, the deficiency of spleen-qi may exhibit symptoms, including poor appetite, dyspepsia, decreased immunity, and even gastrointestinal dysfunction, which are very close to diarrhoea induced by early weaning. Si-jun-zi decoction (SJZD) as traditional prescription for treating spleen-qi deficiency syndrome for thousands of years(Sun et al., 2016; Zheng et al., 2014). Modern medicine also demonstrated that SJZD can regulate intestinal immunity, improve gastrointestinal function and treat diarrhea induced by spleen-qi deficiency. Previous studies have showed that SJZD has various pharmacological activities which related to digestive system(Yu et al., 2014). For instance, the SJZD could prevent and treat the gastric mucosa injury, and inhibit the growth of gastric tumor induced by human sgc-7901 SP cells through promoting apoptosis in rats(Nie et al., 2019). JH Feng et al. has demonstrated that the SJZD can adjust the disorder of gastrointestinal electrical activity and motility of rats with spleen deficiency caused by reserpine(Feng et al., 2010). ZS Zhong et al. has identified that the SJZD could regulate the Cam-MLCK signal pathway, relieve the abnormal tension of gastric smooth muscle and promote gastrointestinal dynamics in rats with spleen deficiency (Zhong et al., 2018). Moreover, the SJZD can significantly reduce the diarrhea rate of piglets by enhancing the cellular and humoral immunity and alleviating weaning stress(Ren et al., 2016).ZH Huang et al. also proved that SJZD could enhance the immunity of small intestine mucosa and promote growing of piglets(Huang, Z. et al., 2017). In order to fit different clinical demands, SJZD was also been modified to owned wider pharmacological actions based on the original major formula and TCM theory,such as Jian-pi Mixture (SJZD + Dioscorea oppositifolia L.(Rhizome)) on Irritable bowel syndrome(Lian et al., 2020), SJZD combined with Astragalus mongholicus Bunge and Dioscorea oppositifolia L. to attenuates colon cancer liver metastasis by increasing macrophage cells(Zhou et al., 2019). However, the protective effect of modified Si-jun-zi granule that combines with Astragalus mongholicus Bunge, Bupleurum chinense DC, Citrus $\times$ aurantium L. and Crataegus pinnatifida Bunge(fruit) to impeded diarrhea induced by earlyweaning in Rex rabbits has not been reported and need for further research. Both Astragalus mongholicus Bunge, Bupleurum chinense DC, Citrus $\times$ aurantium L. and Crataegus pinnatifida Bunge(fruit) were also cited in the TCM formula, which can be used to treat the diarrhea(Che et al., 2019) (Li et al., 2018), and were proved that could improve intestinal structure and immunity(Rahimi and Abdollahi, 2012) (Yu et al., 2018) (Tan et al., 2020).

Previous studies have shown that the protective effect of modified Si-jun-zi granule on diarrhea induced by early-weaning in Rex rabbits was confirmed by growth performance, diarrhea frequency and mortality, but the mechanisms are unclear(LIU et al., 2018). Then, it is necessary to study the potential mechanisms of the protective effect by histomorphology of small intestine, serum biochemical indices including total cholesterol (TC), glutamic pyruvic transaminase (ALT), glutamic oxalacetic transaminase (AST),alkaline phosphatase (ALP) and immunoglobulin (IgA, IgG and IgM) in serum, digestive enzyme activity (lipase, amylase and trypsin), secretory immunoglobulin $A(S \lg A)$ level in the intestinal mucus, intestinal tight junction protein (Zo-1, Claudin-1and Occludin) level and intestinal Glucose transporter (SGLT1 and 
GLUT2) level. Our findings provide new theoretical basis of modified Si-jun-zi granule on enhancing intestinal development and immunity in early-weaned Rex rabbits as dietary supplementation.

\section{Material And Methods}

\section{Modified Si-jun-zi granule}

Modified Si-jun-zi granule (M-SJZG) is constituted of eight herbs, including Codonopsis pilosula (Franch.) Nannf, Atractylodes Lancea (Thunb.) DC, Wolfiporia cocos, Glycyrrhiza uralensis Fisch. ex DC (Preparata), Astragalus mongholicus Bunge, Bupleurum chinense DC, Citrus $\times$ aurantium L. and Crataegus pinnatifida Bunge (fruit) with a dosage ratio of 9:9:9:6:10:6:6:6, all of these were purchased from Sichuan c\&y Traditional Chinese Medicine CO., LTD. and accorded with Chinese veterinary drug code(the details are summarized in Supplementary Table 1). Herbs were mixed and wetted with distilled water $(1: 8, \mathrm{w} / \mathrm{v})$ for $2 \mathrm{~h}$. Then, the mixture was boiled for $3 \mathrm{~h}$ and filtered through multi-layer gauze. The filtrate was gathered and residues were decocted twice with water $(1: 6, w / v)$ for $3 \mathrm{~h}$. At last, the combined filtrates was concentrated to $1.0 \mathrm{~g} / \mathrm{mL}$ which measured by raw medicine. The decoction was mixed with starch (1:1, $\mathrm{v} / \mathrm{w})$. M-SJZG were prepared by wet granulation with proper concentration of alcohol as adhesive for convenience of daily administration.

The quality control of M-SJZG was analyzed using high-performance liquid chromatography (HPLC) with Corona Ultra detection(CAD) in Agilent HPLC system. M-SJZG $(5 \mathrm{~g})$ wasdissolved in $10 \mathrm{~mL}$ Methanol solution (70\%) and diluted 10 times. The solution centrifuged (1500 rpm, $15 \mathrm{~min})$ and filtered $(0.22 \mu \mathrm{m})$, and then, $20 \mu \mathrm{L}$ was injected into an HPLC system for assay. The separation was performed using a reverse-phase column(Intersil ODS-3 $5 \mu \mathrm{m}, 4.6 \mathrm{~mm} \times 250 \mathrm{~mm}$ I.D.) connected with a guard column (C18, $5 \mu \mathrm{m}, 4.6 \mathrm{~mm} \times 10 \mathrm{~mm}$ I.D.). The elution flow rate was $1.0 \mathrm{~mL} / \mathrm{min}$ with a mobile phase gradient of $A-B$ (A: $\mathrm{H}_{2} \mathrm{O} / \mathrm{H}_{3} \mathrm{PO}_{4}=1000 \mathrm{~mL} / 2 \mathrm{~mL} ; \mathrm{B}: \mathrm{CH}_{3} \mathrm{CN}$ ), which was varied as follows:0 min, $90 \% \mathrm{~A}, 10 \% \mathrm{~B} ; 9 \sim 29 \mathrm{~min}$, $90 \sim 85 \% \mathrm{~A}, 10 \sim 15 \% \mathrm{~B} ; 29 \sim 37 \mathrm{~min}, 85 \sim 82 \% \mathrm{~A}, 15 \sim 18 \% \mathrm{~B} ; 37 \sim 46 \min , 82 \% \mathrm{~A}, 18 \% \mathrm{~B} ; 46 \sim 51 \mathrm{~min}, 82$ $\sim 80 \% \mathrm{~A}, 18 \sim 20 \% \mathrm{~B} ; 51 \sim 72 \mathrm{~min}, 80 \sim 72 \% \mathrm{~A}, 20 \sim 28 \% \mathrm{~B} ; 72 \sim 82 \mathrm{~min}, 72 \% \mathrm{~A}, 28 \% \mathrm{~B} ; 82 \sim 87 \mathrm{~min}, 72 \sim$ $70 \% \mathrm{~A}, 28 \sim 30 \% \mathrm{~B} ; 87 \sim 92 \min , 70 \sim 67 \% \mathrm{~A}, 30 \sim 33 \% \mathrm{~B} ; 92 \sim 99 \min , 67 \sim 50 \% \mathrm{~A}, 33 \sim 50 \% \mathrm{~B} ; 99 \sim$ 110 min, 50\% A, 50\% B; $110 \sim 115$ min, $50 \sim 47 \%$ A, 50 53\% B; $115 \sim 118$ min, $47 \sim 30 \%$ A, 53 70\% B; $118 \sim 130 \mathrm{~min}, 30 \%$ A, $70 \%$ B; 0 min, $90 \%$ A, 10\% B; $130 \sim 135$ min, $30 \sim 90 \%$ A, $70 \sim 10 \%$ B; $135 \sim 140$ $\min \varangle 90 \% A, 10 \% B$. The injection volume was $20 \mu \mathrm{L}$, and the UV detection wave length was set at $285 \mathrm{~nm}$ for saikoside A, liquiritin and hesperidin; $250 \mathrm{~nm}$ for glycyrrhizic acid. The fingerprint chromatogram of MSJZG is shown in Supplementary Fig. 1.

\section{Chemicals and reagents}

Glutamine (GIn) was purchased from Huana Chemical Co., Ltd (china), HE stain (Hematoxylin and Eosin) were purchased from Thermo Fisher Co., Ltd (American), all Elisa kits was purchased from Nanjing Jiancheng Bioengineering Institute, FastKing RT kit was purchased from Tiangen Biotech (Beijing) CO., LTD, the others reagents was purchased from ChenDu Chron Chemicals Co., Ltd (china). 
Animals

Two hundred and eighty Rex rabbits, approximately 45 days of age and 750-950g in weight, were obtained from the Rex rabbit institute, Sichuan Academy of Grassland Sciences. The animals were provided with basal diet and tap water at liberty and maintained in cages under controlled conditions $\left(23 \pm 2^{\circ} \mathrm{C}, 12 \mathrm{~h}\right.$ light/dark cycle). All experiments and procedures were carried out according to the Regulations of Experimental Animal Administration issued by the State Committee of Science and Technology of China. The composition and nutrient levels of the basal diet were listed in Table 1.

\section{Growth performance, diarrhea frequency and mortality}

One hundred and twenty Rex rabbits were randomly divided into four groups, including control group and M-SJZG $(0.5 \%, 1 \%, 2 \%)$ groups. Except the control group, M-SJZG were administrated as dietary supplement for thirty consecutive days, to evaluate the protective effect by growth performance, diarrhea frequency $\left(P_{\text {diarrhea }}\right.$ was calculated as equation 1$)$ and mortality.

$$
\mathrm{p}_{\text {diarrhea }}=\frac{\sum A}{\sum B} \times 100 \%
$$

Where A represents the the total times of diarrhea, and B represents the sum of survival days of each rabbit in the group.

\section{Tissue collection and measurement}

To revealed the protective mechanism of M-SJZG from intestinal development and immunity, the others one hundred and sixty rabbits were randomly divided into five groups, including control group, M-SJZG $(0.5 \%, 1 \%, 2 \%)$ groups and Glutamine (GIn) $(0.8 \%)$ group. M-SJZG $(0.5 \%, 1 \%, 2 \%)$ or Gln $(0.8 \%)$ were administrated as a dietary supplement for thirty consecutive days. In each group, the six rabbits were randomly selected, weighted and sacrificed on the 15th and 30th day after treatment, respectively. The intestinal length and relative weight were measured after washing by physiological saline. Then the duodenum, jejunum and ileum were collected and fixed with $4 \%$ paraformaldehyde, respectively.

\section{Histomorphology}

The histopathological changes of various intestinal segments were observed under a high-resolution microscope with photographic facility after being embedded in paraffin and made slides with HE staining. Image pro Plus was used to measure the length of villi and the depth of crypt, then the ratio was calculated as follows (Equation 2).

$$
R_{(V / C)}=\frac{L_{\text {villi }}}{D_{\text {crypt }}}
$$


Where $L_{\text {villi }}$ and $D_{\text {crypts }}$ stand for the length of villi and the depth of crypt, respectively.

\section{ELISA analysis of serum biochemical indices and SIgA}

The serum and intestinal mucus was collected after experimental period. Then the abundance of serum biochemical indices and SIgA on the intestinal mucus were assayed by ELISA kits according to the manufacturer's instructions.

\section{Immunohistochemical analyses of SIgA}

The abundance of SIgA was assessed for paraffin embedded slides after the sections were dewaxed. Endogen biotin and non-specific signals were blocked with appropriated reagents. Antigen retrieval was carried out in a microwave oven (two cycles for 5 min each at $780 \mathrm{~W}$, in citrate buffer, $\mathrm{pH}$ 6.0, twice washed in PBS for 5 min each). Then, the treated slides were overnight incubated with primary antibodies at $-4^{\circ} \mathrm{C}$ in a humidified chamber, washed in PBS, and visualized by biotinylated secondary antibodies followed by stained by DAB kit for 2 min, washed in distilled water. Finally, counterstained with Hematoxylin, dehydrated, transparentized and sealed. At least 10 fields of view from each sample were analyzed with the image analysis software for each protein of interest.

\section{Zo-1, Claudin-1, Occludin, SGLT1 and GLUT2 level assay}

Total RNA was isolated from samples of jejunum, ileum and colon with TRIZOL regent (Tiangen Biotech, Beijing) and then treated with DNase I (Tiangen Biotech, Beijing) according to the manufacturer's instructions. Primers used in this study are presented in Table 2. GAPDH was used as an internal control to normalize expression of target gene transcripts. Real-time PCR was performed as previous studies described(Yin et al., 2015).

\section{Statistical analysis}

The one-way analysis of variance (ANOVA) was used to analyze experimental data by SPSS 25.0 software. All values were presented as the mean $\pm S D$, and $P<0.05$ was considered statistically significant.

\section{Results}

\section{Growth performance}

As shown in Table. 3, the average daily food intake (ADFI) was not significantly affected compared with control group (Table. 3, Pख0.05). Moreover, the feed conversion ratio (FCR) was also significantly decreased after treatment (Table. $3, P<0.05$ ). However, significant improvement in the average daily gain (ADG) was observed after M-SJZG (1.0\% and $2.0 \%)$ was administrated. These results indicated that MSJZG had promoting effects on the growth of early-weaning rabbit. 


\section{Diarrhea frequency and mortality}

The frequency of diarrhea was extremely significantly declined in all treatment groups compared to control group (Table. 4, $\mathrm{P}<0.01)$, and the mortality were observably decreased after M-SJZG $(1.0 \%$ and $2.0 \%$ ) treatment compared with control group (Table 4, $\mathrm{P}<0.01$ ). Furthermore, the effect was dosedependent. These results indicated that M-SJZG had protective effects on diarrhea induced by earlyweaning.

\section{Promotion effect of M-SJZG on the intestinal development in early-weaning Rex rabbits}

The results showed that intestinal length was significantly increased by administrated M-SJZG (2\%) compared to control group (Fig. $1 \mathrm{~A}, \mathrm{P}<0.05)$ at $15 \mathrm{~d}$ and $30 \mathrm{~d}$. Meanwhile, M-SJZG $(0.5 \%, 1.0 \%$ and $2.0 \%)$ and $\mathrm{GIn}(0.8 \%)$ treatment could slightly increase the relative weight of intestine in early-weaning Rex rabbits (Fig. 1B, $P>0.05$ ). Moreover, the ratio of villi length to crypt depth was remarkably risen in jejunum(Fig. $2 B, P<0.05)$ by administrated M-SJZG $(2 \%)$ at 15 d. However, this trend was not significant in duodenum and ileum between experimental period (Fig. 2A, 2C, $P>0.05$ ). (HE staining results and data were listed in Supplementary Fig.2 4. and Supplementary Table.2 5.)

\section{Effects of M-SJZG on serum biochemical indices}

Compared with the control group, the concentration of $\lg A$, IgG and IgM was significantly increased $(\mathrm{P}<$ 0.05 ) by dietary supplementation with $2 \%$ M-SJZG (Fig. 3A), but the activities of ALT and ALP in serum were obviously decreased (Fig. 3 C). Moreover, Supplementation with $0.5,1 \%$ or $2 \%$ M-SJZG significantly decreased $(P<0.05)$ the activity of serum AST (Fig. 3C). Additionally, the serum concentration of TC were significantly reduced $(P<0.05)$ (Fig. 3D).

\section{Effect of M-SJZG on digestive enzyme activity}

Supplementation with $0.5 \%, 1 \%$ or $2 \%$ M-SJZG significantly increased $(P<0.05)$ the activity of lipase, but not amylase and trypsin (Fig. 3B).

\section{Promotion effect of M-SJZG on intestinal mucosal immunity in early-weaning Rex rabbits}

As Elisa results shown in Fig. 4, the concentration of SIgA in intestinal mucus was significantly increased with M-SJZG $(2.0 \%)$ treatment compared to control group $(P<0.05)$ in duodenum and jejunum at $15 \mathrm{~d}$ and 30d. However, the increase of SIgA was not significant in ileum. In addition, the improvement effect was dosage dependence.

The immunohistochemical results of the express of SIgA was shown in Fig 5-7, the positive reaction mainly existed in cytoplasm. The average optical density of the express of SIgA in different groups was measured and shown in Fig 8. The expression of SIgA in jejunum was obviously up-regulation $(P<0.05)$ which soared $10.07 \%$ by high dosage compared with control group, and $8.80 \%$ by Gln treatment. The 
slightly up-regulate $(P>0.05)$ also could be observed in the others treatment groups, including in duodenum and ileum, and shown dosage-dependence.

\section{Intestinal tight junction protein and glucose transporter level}

The results of real-time PCR (Fig. 9-13) showed that in response to M-SJZG or GIn treatment, the expressions of Zo-1, Claudin-1, Occludin, SGLT1 and GLUT2 in intestinal tissues from early weaning Rex rabbits were elevated to high levels. At the end of experimental period, high-dosage treatment significantly $(p<0.05)$ up-regulated the expression of all this proteins mainly in jejunum but SGLT1 in duodenum and ileum.

\section{Discussion}

Early-weaning commonly induced diarrhea in infant animals, even leading to death, often associated with important economic losses for breeding(Nabuurs, 1998). Studies reported that the diarrhea was induced by multiple reasons for early-weaning Rex rabbits(CHEN et al., 2017). On the one hand, the exterior which including infection by virus, bacteria and parasite or intoxication plays the important role for diarrhea(Hodges and Gill, 2010). On the other hand, inner reasons which include underdeveloped digestive system and insufficient nutrients could be the key factors(Keusch et al., 2016). The underdeveloped digestive system was concrete performed in two aspects: the physical and immune barrier, that leads the fragile intestinal track. Clinically studies showed that Gln, peptide growth factors and others, essential nutrients found in breast milk, helping digestive system development(Martin et al., 2016). Therefore, the early weaning reduced the intake of essential nutrition. Based on the discussion above, the promotion of intestinal development was not only beneficial in the establishment of barriers but also had the protective effect on diarrhea induced by early weaning.

SJZD, a Chinese herbal decoction consisting of Codonopsis pilosula, Atractylodes lancea, Tuckahoe and Glycyrrhiza uralensis, is widely used to nourish deficiency of spleen and stomach qi in China 800 years ago and is still widely used to improve immunity and digestive function disorders(Li et al., 2017). In recent decades, SJZD was modified to broaden the range of application. JingMing Chen et al. Combined SJZD with Hedysarum polybotrys and Ligustrum lucidum, to impede cachexia(Chen et al., 2019). GuihuaTian et al. researched the therapeutic effect of modified SJZD to chronic atrophic gastritis(Tian et al., 2019). In our study, the Astragalus mongholicus Bunge, Bupleurum chinense, Citrus $x$ aurantium and Crataegus pinnatifida Bunge (fruit) were added to SJZD and prepared into granule as dietary supplement, for treating diarrhea induced by early weaning. Zhibo Guan et al.(Guan et al., 2018) has verified the effective constituents of Si-jun-zi decoction mainly included ginsenosides, flavonoids and polysaccharides with Fourier transform ion cyclotron resonance mass spectrometry. It has been proved that polysaccharides in SJZD has nourishment function and a variety of pharmacological effects(Huang, C. et al., 2017; Wang et al., 2016). Therefore, SJZD/M-SJZG could be the supplements for early weaning Rex rabbits growth and development, and help them to conform non-breast feeding. 
Growth performance, diarrhea frequency and mortality were first assessed after early weaning and MSJZG treatment. Our data showed that M-SJZG significantly improvement growth performance, ADG and FCR. As we know, early weaning can affect the development of digestive system and induced some disorders, which can lead to diarrhea and even death. However, M-SJZG had noticeable effects on protection of it. This results indicated that M-SJZG might improve the survival rate from early weaning(Delgado et al., 2019). Previous studies have shown that dietary supplement such as GIn (positive group) could improve intestinal development and protect it from atrophy and injury under various stress conditions in young animals(Wang et al., 2015). The effects of M-SJZG were evaluated from the length and relative weight of intestine in early weaning Rex Rabbits that were always used to assessed intestinal development level in previous studies(Fung et al., 2016). Intestine is one of the most important organs in the digestive system, whether its structure and function are normal or not can affect the digestion-absorption function. The length and relative weight of the intestine was found increased compared to control group after M-SJZG treatment. The ratio of villus height and crypt depth also as an important index, which in direct proportion to intestinal digestion and absorption capacity(Liu et al., 2019). The results shown that M-SJZG treatment has the dosage-dependence for the ratio increasing, and only high dose (2.0\%) M-SJZG administrated has the similar effect to positive control $(0.8 \% \mathrm{Gln})$.

A salient observation from the present study is M-SJZG supplementation reduced oxidative damage in the liver, as shown by oxidative damage and health markers reduction (including serum ALT, AST and ALP). Serum TC is mainly synthesized by the liver, as the structural component of cells, also can be converted into vitamin D3 to regulate calcium and phosphorus metabolism, promote intestinal calcium and phosphorus absorption and calcification. In present test, Serum TC reduction and the increased lipase activity marked the promotion of lipid metabolism and transformation. Therefore M-SJZG supplementation improved the growth performance can be partly attributed to the lipid metabolism. A series of serum immunoglobulin (IgA, IgG and $\lg M$ ) are important indexes to measure the humoral immunity of the body. Serotype IgA is produced mainly by plasma cells in mesenteric lymphoid tissue. The concentration increase of immunoglobulin, especially serotype IgA, revealed the decrease of diarrhea frequency and mortality may be related to humoral and gut immunity.

The mucus of adjacent to the epithelial cells in the gastrointestinal tract constitutes an immunity barrier, thereby providing a layer to against infection, in which SIgA was the mainly antibody(Perez-Lopez et al., 2016). The early weaning induced diarrhea not only related to intestinal development but also to pathogenic bacterium infection. This study assessed the SIgA level in intestinal mucus by Elisa. The results illustrated that the content of SIgA was increased compared with control group through M-SJZG administrated, and it can be observed that the significantly promoting mainly focused on the first stage of administration $(0 \sim 15 d)$ which was lined with the tendency of $R_{(V / C)}$. This phenomenon could be attributed to the period of intestinal development and the function of SIgA(Gidenne and Fortun-Lamothe, 2002). Recently, G. P. Donaldson and his cooperators manifested gut microbiota utilize immunoglobulin $A$ for mucosal colonization, which can improve intestinal adaptability to outside(Donaldson et al., 2018). Therefore, the SIgA for intestinal development was satisfied by production in the first stage of 
administration $(0 \sim 15 \mathrm{~d})$. Then, the expression of SIgA in intestine was estimated by immunohistochemical staining, and the result also demonstrated M-SJZG treatment can increase it, to a certain extent.

The epithelial surface of the gastrointestinal tract is also a physical barrier against the "outside". The permeability and the absorption function were closely related to the intestinal tight junction proteins (Zo1, Claudin1, Occludin) and the glucose transporter (SGLT1 and GLUT2), respectively. The expression increase of all these proteins by M-SJZG treatment, was mainly reflected in jejunum which was corresponded with jejunum physiological function, digestion and absorption.

Based on the discussion above, the protective effects of M-SJZG on diarrhea induced by early-weaning in Rex rabbits could be attributed to the improvement of intestinal development and barriers establishment.

\section{Conclusion}

In this study, the protective effects of M-SJZG on diarrhea induced by early-weaning were examined in Rex rabbits. The main achievements of our study are as follows: (a) M-SJZG can promote the intestinal development and improve morphology and function. The optimal dose is $2 \%$ for early-weaned (45d) Rex rabbits as a dietary supplement. (b) The intestine was improved by dietary supplementation of $2 \% \mathrm{M}$ SJZG or $0.8 \%$ Gln. However, The $2 \%$ M-SJZG is better for enhancing intestinal glucose absorption. (c) Modern pharmacological thoughts and methods were combined with the ancient theories of traditional Chinese medicine to delve the protective mechanism of M-SJZG from the gene and protein levels, which laid a theoretical foundation for the clinical use of M-SJZG.

\section{Abbreviations}

ALT: glutamic pyruvic transaminase;

ALP: alkaline phosphatase

AST: glutamic oxalacetic transaminas;

GIn: Glutamine;

GLUT2: recombinant Glucose transporter 2;

IgA: immunoglobulin A;

IgG: immunoglobulin G;

IgM: immunoglobulin $\mathrm{M}$;

M-SJZG: modified Si-jun-zi granule; 
SJZD: Si-jun-zi decoction;

SIgA: secretory immunoglobulin A;

SGLT1: re-combinant Sodium/Glucose co-transporter 1;

TC: total cholesterol

TCM: traditional Chinese medicine

Zo-1: zonula occludens-1;

\section{Declarations}

\section{Ethics approval and consent to participate}

All animal procedures were in accordance with the national standard, Laboratory Animal-Requirements of Environment and Housing Facilities (GB14925-2001), the Sichuan Agricultural University Institutional Animal Care and Use Committee under permit number CSQ-2018203003.

\section{Consent for publication}

Not applicable.

\section{Availability of data and material}

The datasets produced and/or analyzed during the current study are available from the corresponding author on reasonable request.

\section{Competing interests}

The authors report no conflicts of interest.

\section{Funding}

This work was financially supported by the Earmarked Fund for the China Agriculture Research System (CARS-43-A-3) \the Science \& Technology Department of Sichuan Province (2016NYZ0046)and the basic scientific research operating expenses of Sichuan Science \& Technology Department.

\section{Author contributions}

Shiqi Chen $₫$ Methodology, Data Curation, Writing-Original draft preparation.

Ning Liu: Conceptualization, Investigation, Formal analysis.

Dongbo Li: Visualization, Validation. 
Haibo Dou: Visualization.

Quanjin Liu: Validation.

Hanzhong Liu: Visualization.

Ping Wang: Supervision.

Zhiju Yu: Software.

Gang Shu, Juchun Lin: Supervision.

Wei Zhang, Huaqiao Tang: Software.

Guangneng Peng: Writing-Reviewing \& Editing.

Kai Zhang: Writing-Review \& Editing.

Bin Wen: Writing-Review \& Editing.

Ling Zhao: Writing-Review \& Editing.

Hualin Fu: Project administration, Funding acquisition,Writing-Reviewing \& Editing

\section{Acknowledgements}

Not applicable.

\section{References}

1. Che, D., Adams, S., Wei, C., Gui-Xin, Q., Atiba, E.M., Hailong, J., 2019. Effects of Astragalus membranaceus fiber on growth performance, nutrient digestibility, microbial composition, VFA production, gut $\mathrm{pH}$, and immunity of weaned pigs. MICROBIOLOGYOPEN 8(5), e00712.

2. CHEN, H.-J., YANG, W.-Y., WANG, C.-Y., 2017. The Review on Structure of Intestinal Flora at Different Growth Stages of Rabbits. DEStech Transactions on Biology and Health(icmsb).

3. Chen, J.-M., Yang, T.-T., Cheng, T.-S., Hsiao, T.-F., Chang, P.M.-H., Leu, J.-Y., Wang, F.-S., Hsu, S.-L., Huang, C.-Y.F., Lai, J.-M., 2019. Modified Sijunzi decoction can alleviate cisplatin-induced toxicity and prolong the survival time of cachectic mice by recovering muscle atrophy. J. Ethnopharmacol. 233, 47-55.

4. Delgado, R., Abad-Guamán, R., Nicodemus, N., Diaz-Perales, A., García, J., Carabaño, R., Menoyo, D., 2019. Effect of pre-and post-weaning dietary supplementation with arginine and glutamine on rabbit performance and intestinal health. BMC Vet. Res. 15(1), 199.

5. Donaldson, G., Ladinsky, M., Yu, K., Sanders, J., Yoo, B., Chou, W.-C., Conner, M., Earl, A., Knight, R., Bjorkman, P., 2018. Gut microbiota utilize immunoglobulin A for mucosal colonization. Science 
360(6390), 795-800.

6. Feng, J., Shang, H., Ren, P., Huang, X., Ju, L., 2010. Effects of SiJunzi Tang on gastroduodenal myoeletrical activity of rat spleen insufficiency syndrome model. J Logistics University PAP(Medical Sciences) 019(1), 8-9,56.

7. Fung, C.M., White, J.R., Brown, A.S., Gong, H., Weitkamp, J.-H., Frey, M.R., McElroy, S.J., 2016. Intrauterine growth restriction alters mouse intestinal architecture during development. PLoS One 11(1), e0146542.

8. Gidenne, T., Fortun-Lamothe, L., 2002. Feeding strategy for young rabbits around weaning: a review of digestive capacity and nutritional needs. Animal Science 75(2), 169-184.

9. Guan, Z., Wang, M., Cai, Y., Yang, H., Zhao, M., Zhao, C., 2018. Rapid characterization of the chemical constituents of Sijunzi decoction by UHPLC coupled with Fourier transform ion cyclotron resonance mass spectrometry. J. Chromatogr. B 1086, 11-22.

10. Hodges, K., Gill, R., 2010. Infectious diarrhea: cellular and molecular mechanisms. Gut microbes 1(1), 4-21.

11. Huang, C., Zhu, Z., Cao, X., Chen, X., Fu, Y., Chen, Z., Li, L., Song, X., Jia, R., Yin, Z., 2017. A Pectic Polysaccharide from Sijunzi Decoction Promotes the Antioxidant Defenses of SW480 Cells. Molecules 22(8), 1341.

12. Huang, Z., Wu, J., Ren, D., Cao, C., 2017. Effect of supplementary Sijunzi decoction on levels of serum immune factors and intestinal mucosa immunity of piglets. J Northwest A\&F University(Natural Science Edition) 045(003), 75-81.

13. Keusch, G.T., Walker, C.F., Das, J.K., Horton, S., Habte, D., 2016. Diarrheal diseases.

14. Kumar, A., Kamboj, M., Kumar, S., Jingar, S.C., Meena, R., Yadav, M., Lawania, P., Bugalia, H., Jatolia, P., 2017. Influence of Calf Weaning at Birth on Reproductive Performance of Murrah Buffaloes. Int. J. Curr. Microbiol. App. Sci 6(11), 4238-4241.

15. Li, J., Cui, H., Cai, Y., Lin, J., Song, X., Zhou, Z., Xiong, W., Zhou, H., Bian, Y., Wang, L., 2018. Tong-XieYao-Fang Regulates 5-HT Level in Diarrhea Predominant Irritable Bowel Syndrome Through Gut Microbiota Modulation. Front. Pharmacol. 9, 1110.

16. Li, L., Zou, C., Zhou, Z., Yu, X., 2017. Effects of herbal medicine Sijunzi decoction on rabbits after relieving intestinal obstruction. Braz. J. Med. Biol. Res. 50(11).

17. Lian, Q., Ding, H., Zhu, H., Zhang, C., Yu, S., Jie, H., Zhang, Y., Wu, B., Liang, G., Zhang, G., 2020. Study of Jianpi Mixture on Intestinal Microbiota of Diarrhea Irritable Bowel Syndrome Mice. Evid. Based Complement. Alternat. Med. 2020.

18. Liu, L., Zuo, W., Li, F., 2019. Dietary addition of Artemisia argyi reduces diarrhea and modulates the gut immune function without affecting growth performances of rabbits after weaning. J. Anim. Sci. 97(4), 1693-1700.

19. LIU, N., Du, D., Xu, C., 2018. Effects of Different Chinese Herbal Compound on Growth Performance and Serum biochemical Indexes of Wean Rax Rabbits. China Animal Husbandry \& Veterinary Medicine 045(002), 422-428. 
20. Liu, S.-H., Chuang, W.-C., Lam, W., Jiang, Z., Cheng, Y.-C., 2015. Safety surveillance of traditional Chinese medicine: current and future. Drug Saf. 38(2), 117-128.

21. Martin, C.R., Ling, P.-R., Blackburn, G.L., 2016. Review of infant feeding: key features of breast milk and infant formula. Nutrients 8(5), 279.

22. Nabuurs, M., 1998. Weaning piglets as a model for studying pathophysiology of diarrhea. Vet. Q. 20(sup3), 42-45.

23. Nie, S., Zhao, Y., Qiu, X., Wang, W., Yao, Y., Yi, M., Wang, D., 2019. Metabolomic Study on Nude Mice Models of Gastric Cancer Treated with Modified Si Jun Zi Tang via HILIC UHPLC-Q-TOF/MS Analysis. Evid. Based Complement. Alternat. Med. 2019.

24. Perez-Lopez, A., Behnsen, J., Nuccio, S.-P., Raffatellu, M., 2016. Mucosal immunity to pathogenic intestinal bacteria. NAT REV IMMUNOL 16(3), 135-148.

25. Rahimi, R., Abdollahi, M., 2012. Herbal medicines for the management of irritable bowel syndrome: a comprehensive review. World journal of gastroenterology: WJG 18(7), 589.

26. Ren, D., He, S., Cao, C., Dong, J., Fan, M., Huang, Z., Li, Y., Wang, X., Wu, J., 2016. Effect of supplementary Sijunzi decoction on digestive and absorptive functions and EGF expression of small intestine in piglets. Chinese Veterinary Science 046(8), 1065-1072.

27. Richter, V., Lebl, K., Baumgartner, W., Obritzhauser, W., Käsbohrer, A., Pinior, B., 2017. A systematic worldwide review of the direct monetary losses in cattle due to bovine viral diarrhoea virus infection. The Veterinary Journal 220, 80-87.

28. Sun, J., Zhang, L., He, Y., Zhang, K., Wu, L., Fan, Y., Xie, Z., 2016. To unveil the molecular mechanisms of qi and blood through systems biology-based investigation into Si-Jun-Zi-Tang and Si-Wu-Tang formulae. Sci. Rep. 6, 34328.

29. Szajewska, H., Canani, R.B., Guarino, A., Hojsak, I., Indrio, F., Kolacek, S., Shamir, R., Vandenplas, Y., van Goudoever, J.B., Weizman, Z., 2016. Probiotics for the prevention of antibiotic-associated diarrhea in children. J. Pediatr. Gastroenterol. Nutr. 62(3), 495-506.

30. Tan, X., Sun, Z., Zhou, M., Zou, C., Kou, H., Vijayaraman, S.B., Huang, Y., Lin, H., Lin, L., 2020. Effects of dietary hawthorn extracts supplementation on lipid metabolism, skin coloration and gut health of golden pompano (Trachinotus ovatus). Aquaculture, 734921.

31. Tian, G., Wu, C., Li, J., Liang, B., Zhang, F., Fan, X., Li, Z., Wang, Y., Li, Z., Liu, D., 2019. Network pharmacology based investigation into the effect and mechanism of Modified Sijunzi Decoction against the subtypes of chronic atrophic gastritis. Pharmacol. Res. 144, 158-166.

32. Vicario, M., González-Castro, A.M., Martínez, C., Lobo, B., Pigrau, M., Guilarte, M., de Torres, I., Mosquera, J.L., Fortea, M., Sevillano-Aguilera, C., 2015. Increased humoral immunity in the jejunum of diarrhoea-predominant irritable bowel syndrome associated with clinical manifestations. Gut 64(9), 1379-1388.

33. Wang, B., Wu, G., Zhou, Z., Dai, Z., Sun, Y., Ji, Y., Li, W., Wang, W., Liu, C., Han, F., 2015. Glutamine and intestinal barrier function. Amino Acids 47(10), 2143-2154. 
34. Wang, R., Peng, Y., Meng, H., Li, X., 2016. Protective effect of polysaccharides fractions from Sijunzi decoction in reserpine-induced spleen deficiency rats. RSC Advances 6(65), 60657-60665.

35. Wischmeyer, P.E., McDonald, D., Knight, R., 2016. Role of the microbiome, probiotics, and 'dysbiosis therapy'in critical illness. Curr. Opin. Crit. Care 22(4), 347.

36. Wood, K., Palmer, S., Steele, M., Metcalf, J., Penner, G., 2015. The influence of age and weaning on permeability of the gastrointestinal tract in Holstein bull calves. J. Dairy Sci. 98(10), 7226-7237.

37. Yarnall, M.J., Thrusfield, M.V., 2017. Engaging veterinarians and farmers in eradicating bovine viral diarrhoea: a systematic review of economic impact. The Veterinary Record 181(13), 347.

38. Yin, J., Wu, M., Duan, J., Liu, G., Cui, Z., Zheng, J., Chen, S., Ren, W., Deng, J., Tan, X., 2015. Pyrrolidine dithiocarbamate inhibits NF-KappaB activation and upregulates the expression of Gpx1, Gpx4, occludin, and ZO-1 in DSS-induced colitis. Appl. Biochem. Biotechnol. 177(8), 1716-1728.

39. Yu, X., Cui, Z., Zhou, Z., Shan, T., Li, D., Cui, N., 2014. Si-Jun-Zi decoction treatment promotes the restoration of intestinal function after obstruction by regulating intestinal homeostasis. Evid. Based Complement. Alternat. Med. 2014.

40. Yu, X., Sun, S., Guo, Y., Liu, Y., Yang, D., Li, G., Lü, S., 2018. Citri Reticulatae Pericarpium (Chenpi): Botany, ethnopharmacology, phytochemistry, and pharmacology of a frequently used traditional Chinese medicine. J. Ethnopharmacol. 220, 265-282.

41. Zheng, X.-f., Tian, J.-s., Liu, P., Xing, J., Qin, X.-m., 2014. Analysis of the restorative effect of Bu-zhongyi-qi-tang in the spleen-qi deficiency rat model using $1 \mathrm{H}-\mathrm{NMR}$-based metabonomics. J. Ethnopharmacol. 151(2), 912-920.

42. Zhong, Z., Zhang, H., Zhang, W., He, G., Ye, Z., Wang, J., Huang, S., 2018. Effect of Si Junzitang on CaM-MLCK Pathway in Rats with Spleen Deficiency and Gastrointestinal Motility Disorder. Chinese J Experimental TMF 024(005), 5.

43. Zhou, J.-Y., Chen, M., Wu, C.-E., Zhuang, Y.-W., Chen, Y.-G., Liu, S.-L., 2019. The modified Si-Jun-Zi Decoction attenuates colon cancer liver metastasis by increasing macrophage cells. BMC Complement. Altern. Med. 19(1), 86.

44. Zhu, Y., Wang, C., Li, F., 2015. Impact of dietary fiber/starch ratio in shaping caecal microbiota in rabbits. Can. J. Microbiol. 61(10), 771-784.

\section{Tables}


Table 1.

The composition and nutrient levels of basal diet (air-dry basis) \%

\begin{tabular}{|c|c|c|c|}
\hline Items & Content & Nutrient Levels & Content \\
\hline Ingredients & & Digestible Energy $\varangle \mathrm{DE}, \mathrm{MJ} / \mathrm{kg} \rrbracket$ & 10.21 \\
\hline Corn & 20.0 & Crude Protein(CP) & 16.00 \\
\hline Bran & 22.8 & Crude Fibre (CF) & 14.29 \\
\hline Peanut vine & 40.1 & $\mathrm{EE}$ & 3.02 \\
\hline Soyben meal & 14.6 & Met+Cys & 0.46 \\
\hline $\mathrm{NaCl}$ & 0.5 & Lys & 0.56 \\
\hline $\mathrm{CaHPO}_{4}$ & 1.0 & $\mathrm{Ca}$ & 0.58 \\
\hline Premix & 1.0 & $\mathrm{P}$ & 0.62 \\
\hline Total & 100 & I $\triangle \mathrm{mg} / \mathrm{kg} \rrbracket$ & 0.22 \\
\hline
\end{tabular}

(1) The premix provided following per kilogram of diet: Lys 1.5 g; VA 8000 IU; VD 31000 IU; VE 50 mg; Fe 100 mg; Cu 50 mg; Mg 150 mg; Zn 50 mg; Mn 30 mg; Se 0.1 mg

(2)Nutirent levels were calculated values. 
Table 2 .

Primer sequences for qPCR

\begin{tabular}{|c|c|c|c|c|c|}
\hline Gene & Geneback & \multicolumn{2}{|c|}{ Primer sequences ( $5^{\prime}$ to3') } & bp & Temperature ${ }^{\circ} \mathrm{C}$ \\
\hline \multirow[t]{2}{*}{ Claudin1 } & \multirow[t]{2}{*}{ DQ_993356 } & $\mathrm{F}$ & GGAAGATGATGAGGAGCAA & \multirow[t]{2}{*}{77} & \multirow[t]{2}{*}{$59^{\circ} \mathrm{C}$} \\
\hline & & $\mathrm{R}$ & AGCCCAGCCAGTGAAAA & & \\
\hline \multirow[t]{2}{*}{ Occludin } & \multirow[t]{2}{*}{ XM_008262318 } & $\mathrm{F}$ & СTTGCCTGGGACAGAACCTA & \multirow[t]{2}{*}{121} & \multirow[t]{2}{*}{$59^{\circ} \mathrm{C}$} \\
\hline & & $\mathrm{R}$ & AGCCATAACCGTAGCCGTAA & & \\
\hline \multirow[t]{2}{*}{ Zo1 } & \multirow[t]{2}{*}{ XM_008269782 } & $\mathrm{F}$ & GACTGATGCGAAGACGTTGA & \multirow[t]{2}{*}{117} & \multirow[t]{2}{*}{$59^{\circ} \mathrm{C}$} \\
\hline & & $\mathrm{R}$ & GCAGAATGGATGCTGTCAGA & & \\
\hline \multirow[t]{2}{*}{ SGLT1 } & \multirow[t]{2}{*}{ EU_414633 } & $\mathrm{F}$ & TGTTCCGCAGGGACACTAA & \multirow[t]{2}{*}{75} & \multirow[t]{2}{*}{$59^{\circ} \mathrm{C}$} \\
\hline & & $\mathrm{R}$ & GGGATCAGGACGTAAAGAGG & & \\
\hline \multirow[t]{2}{*}{ GLUT2 } & \multirow[t]{2}{*}{ XM_01734 } & $\mathrm{F}$ & AGGCACTGTCCACCACC & \multirow[t]{2}{*}{161} & \multirow[t]{2}{*}{$59^{\circ} \mathrm{C}$} \\
\hline & & $\mathrm{R}$ & GTCTCCAAGCCACCCAC & & \\
\hline \multirow[t]{2}{*}{ GAPDH } & \multirow[t]{2}{*}{ NM_001082253 } & $\mathrm{F}$ & TGCCAСССАСТССТСТА & \multirow[t]{2}{*}{163} & \multirow[t]{2}{*}{$59^{\circ} \mathrm{C}$} \\
\hline & & $\mathrm{R}$ & AGTAAGAGCCCTCAAACCACCGG & & \\
\hline
\end{tabular}

Table 3.

Effects of M-SJZG on growth performance of early-weaning Rex rabbits

\begin{tabular}{|lllll|}
\hline index & control group & $0.5 \%$ & $1.0 \%$ & $2.0 \%$ \\
\hline $\mathrm{ADFl} /(\mathrm{g} / \mathrm{d})$ & $90.27 \pm 2.34$ & $90.47 \pm 2.43$ & $91.31 \pm 2.76$ & $91.72 \pm 0.78$ \\
\hline $\mathrm{ADG} /(\mathrm{g} / \mathrm{d})$ & $14.95 \pm 4.50$ & $18.28 \pm 1.31$ & $19.01 \pm 2.89 *$ & $19.29 \pm 3.27 *$ \\
\hline $\mathrm{FCR}$ & $6.53 \pm 2.01$ & $4.97 \pm 0.42 *$ & $4.89 \pm 0.69 *$ & $4.86 \pm 0.74 *$ \\
\hline
\end{tabular}

${ }^{*} \mathrm{p}<0.05$ vs the control group

Table 4.

Effects of M-SJZG on diarrhea rate and mortality rate ofearly-weaning Rex rabbits

\begin{tabular}{|lllll|}
\hline index & control group & $0.5 \%$ & $1.0 \%$ & $2.0 \%$ \\
\hline Diarrhea rate/\% & $18.52 \pm 4.96$ & $9.76 \pm 2.80^{\star * *}$ & $4.79 \pm 2.16^{\star * *}$ & $1.67 \pm 0.91^{\star *}$ \\
\hline Mortality/\% & $13.30 \pm 10.33$ & $10.00 \pm 2.95$ & $6.67 \pm 2.33^{* *}$ & $1.90 \pm 0.50$ ** \\
\hline
\end{tabular}


${ }^{*} \mathrm{p}<0.05$ and ${ }^{* *} \mathrm{p}<0.01$ compared with control group.

\section{Figures}
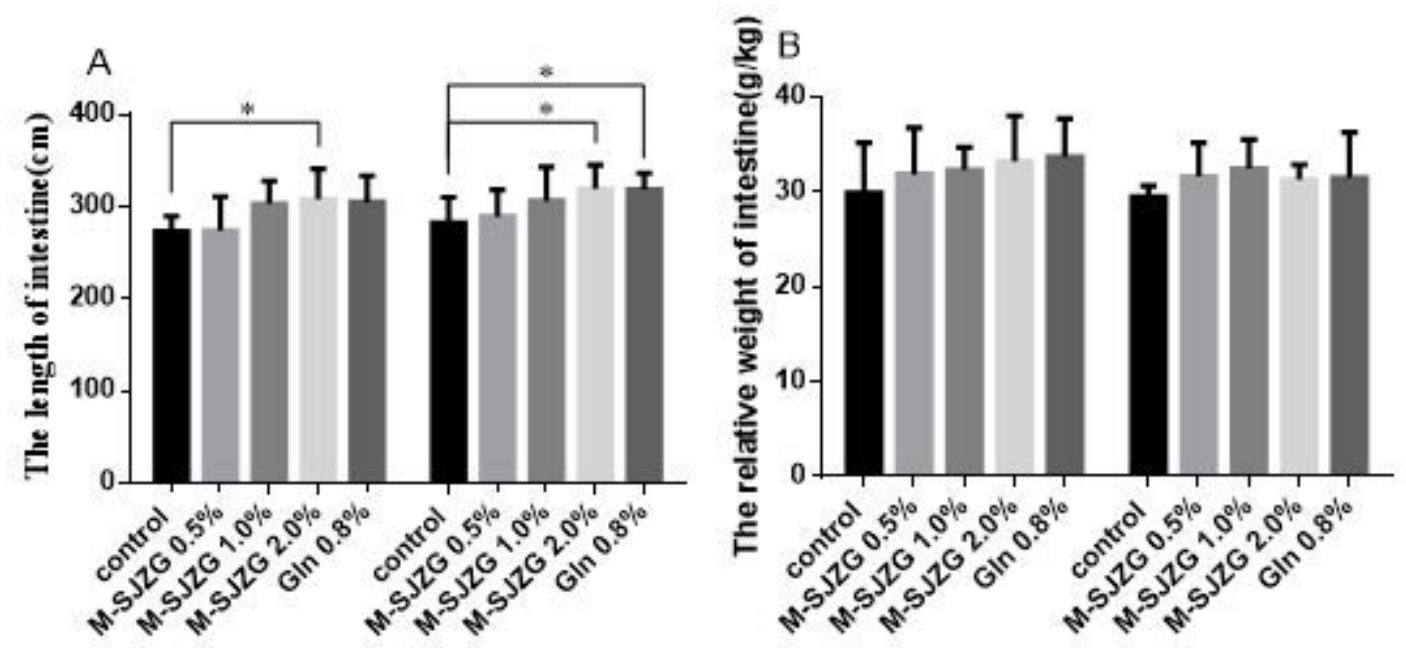

Figure 1

Effects of M-SJZG on intestinal length $(A)$ and relative weight $(B)$ in early-weaning Rex rabbits. Data are expressed as mean $\pm S D$. $(n=6),{ }^{*} P<0.05$. 

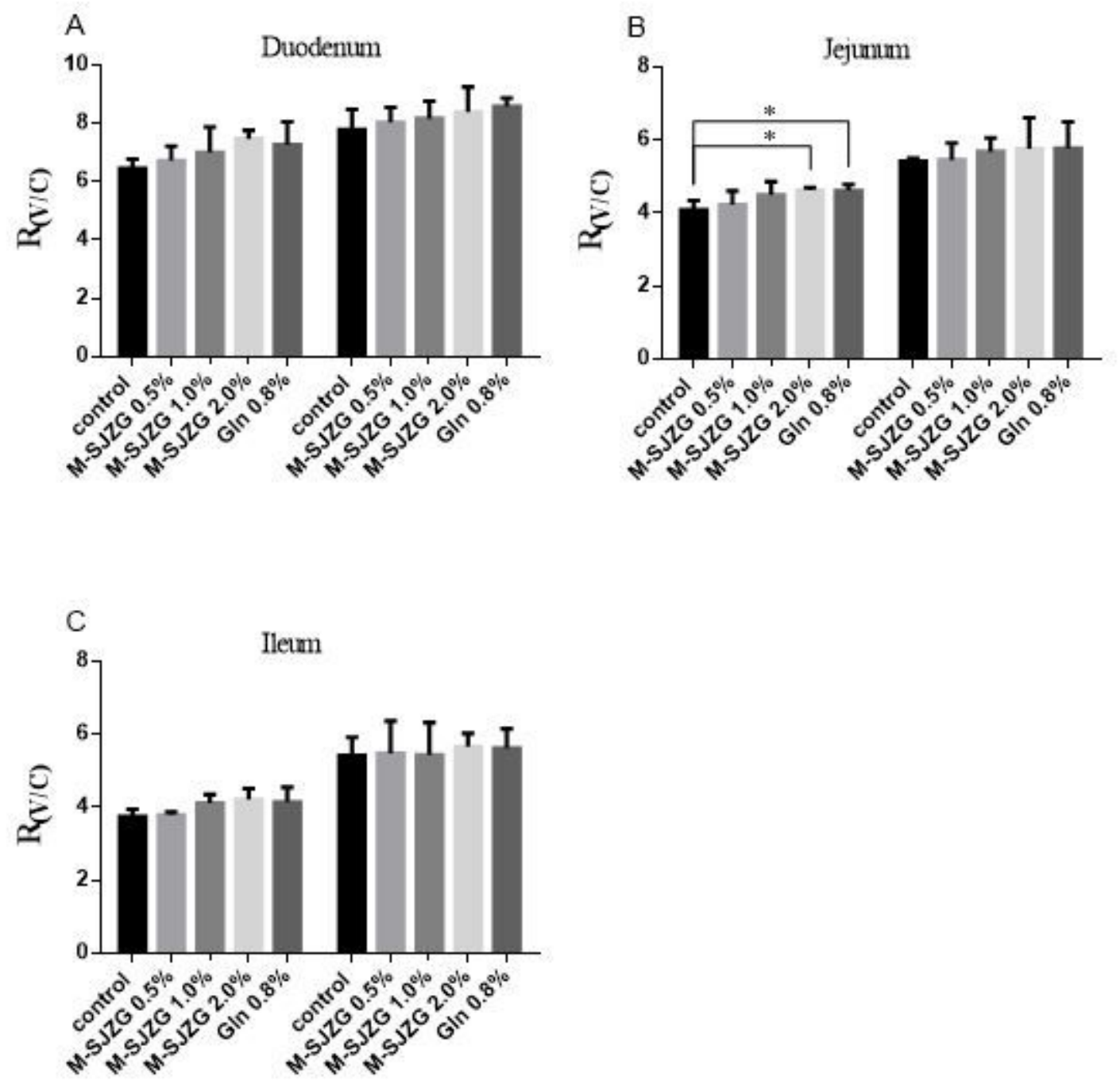

Figure 2

Effects of M-SJZG on the ratio of villi length to crypt depth in duodenum (A), jejunum (B) and ileum (C) in early-weaning Rex rabbits. Data are expressed as mean $\pm S D$. $(n=6),{ }^{*}<0.05$. 

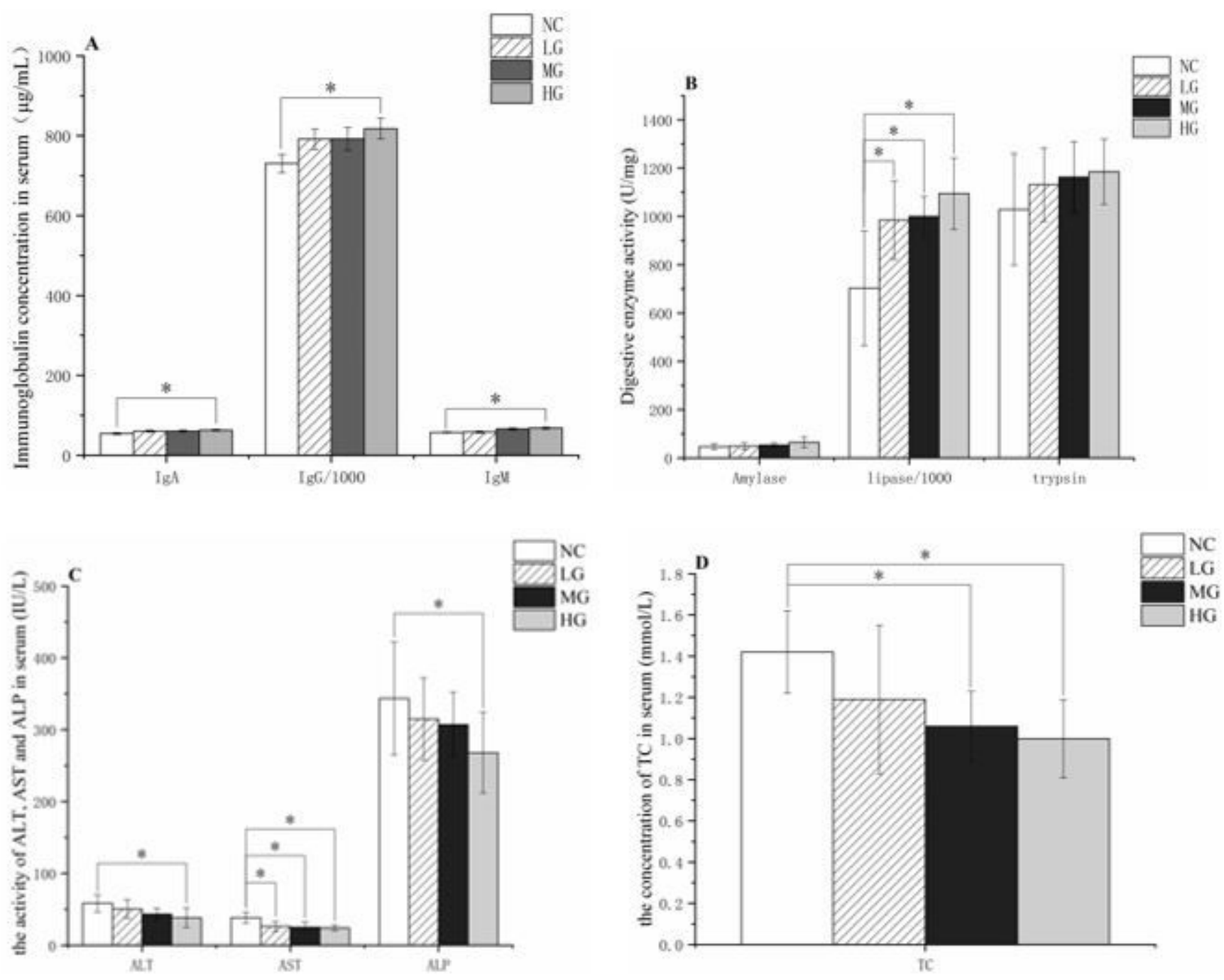

\section{Figure 3}

Serum biochemical indices after treatment, the concentration of immunoglobulin in the serum (A); the activity of digestive enzyme (B); the concentration of ALT, AST and ALP in the serum (C); the concentration of TC in the serum (D). * $p<0.05$ compared with NC. NC, control group; LD, $0.5 \%$ M-SJZG group; MD, 1.0\%M-SJZG group; HD,2.0\% M-SJZG group; 

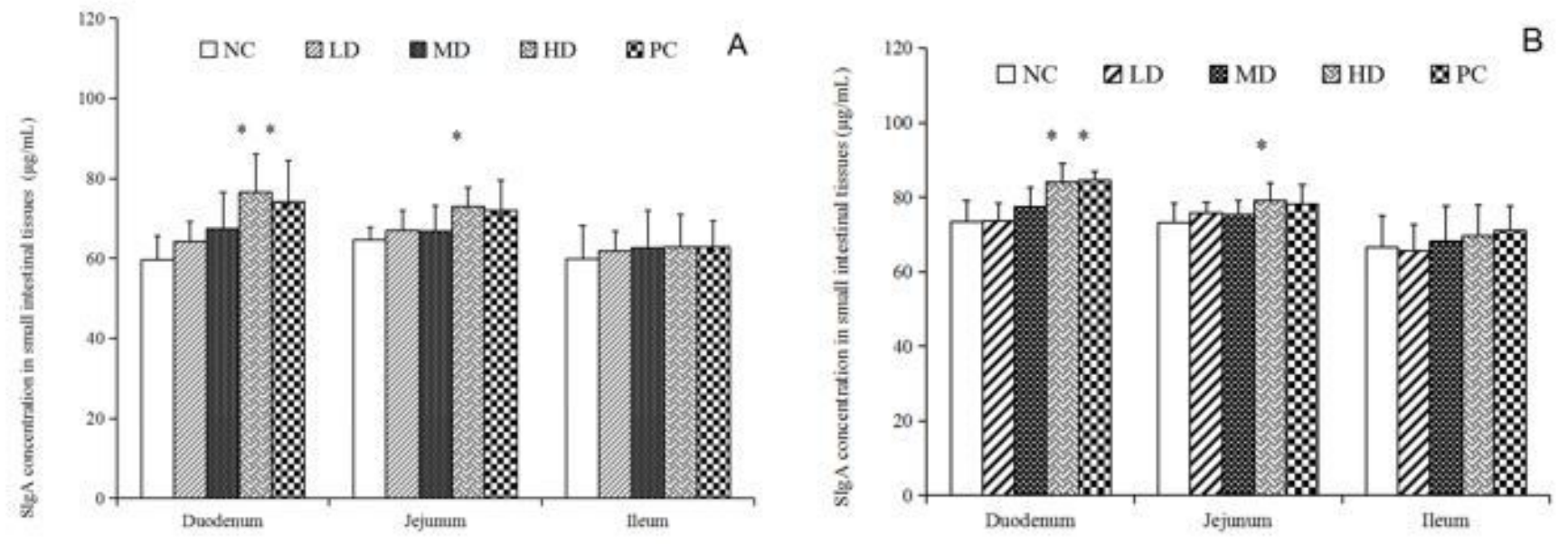

\section{Figure 4}

SlgA content in small intestinal tissues by Elisa, 0 15d (A) and 15 30d (B) $(n=6),{ }^{*}<<0.05$ compared with NC. NC, control group; LD, 0.5\% M-SJZG group; MD, 1.0\%M-SJZG group; HD,2.0\% M-SJZG group; PC, positive, $0.8 \%$ Gln group.
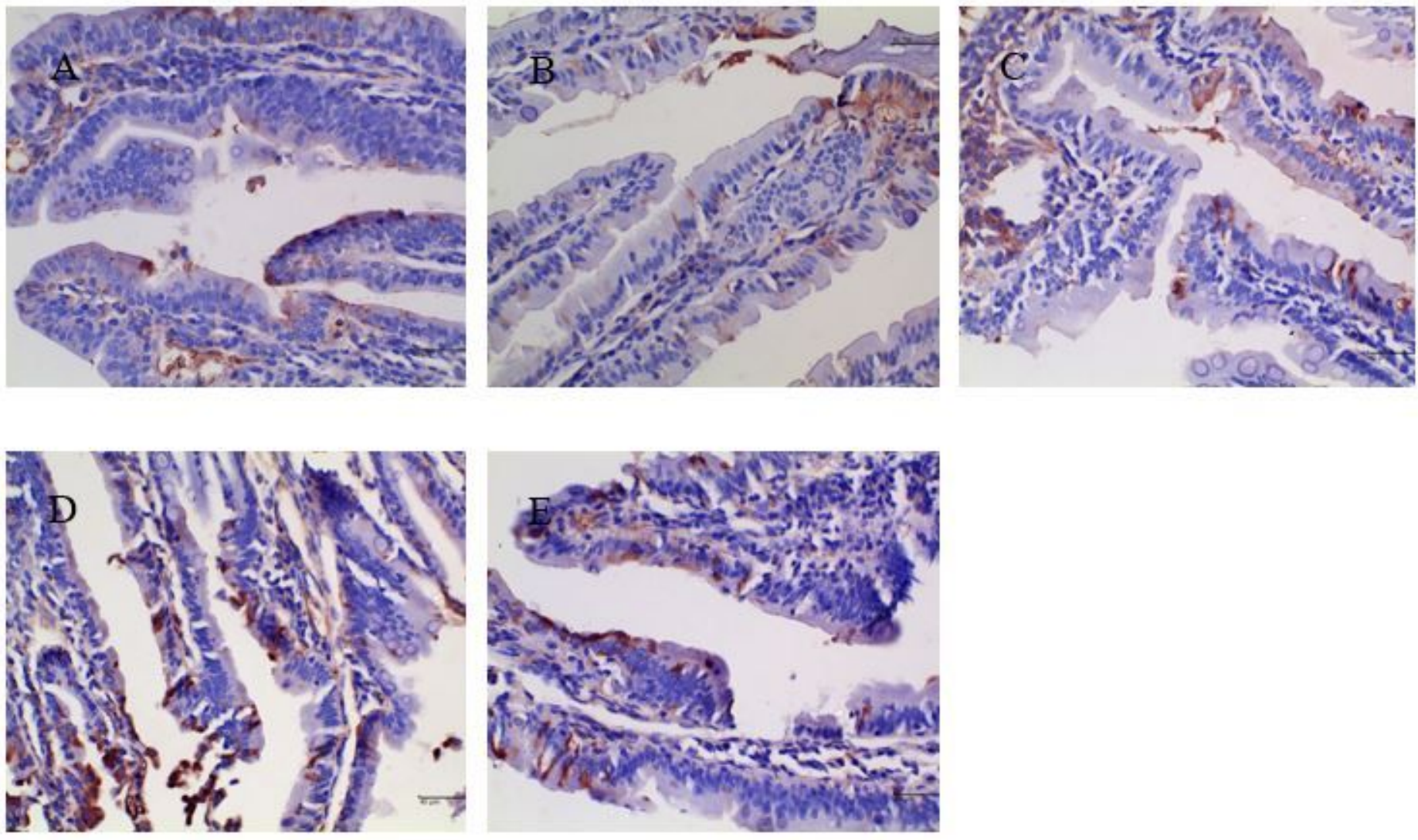

Figure 5 
Immunohistochemical detection of SIgA of duodenum intestinal mucosa different groups $\varangle 0 \sim 30 \mathrm{~d} \nabla(\times 400)$. The negative and positive cells were blue and brown, respectively. The positive reaction was mainly operated in cytoplasm.
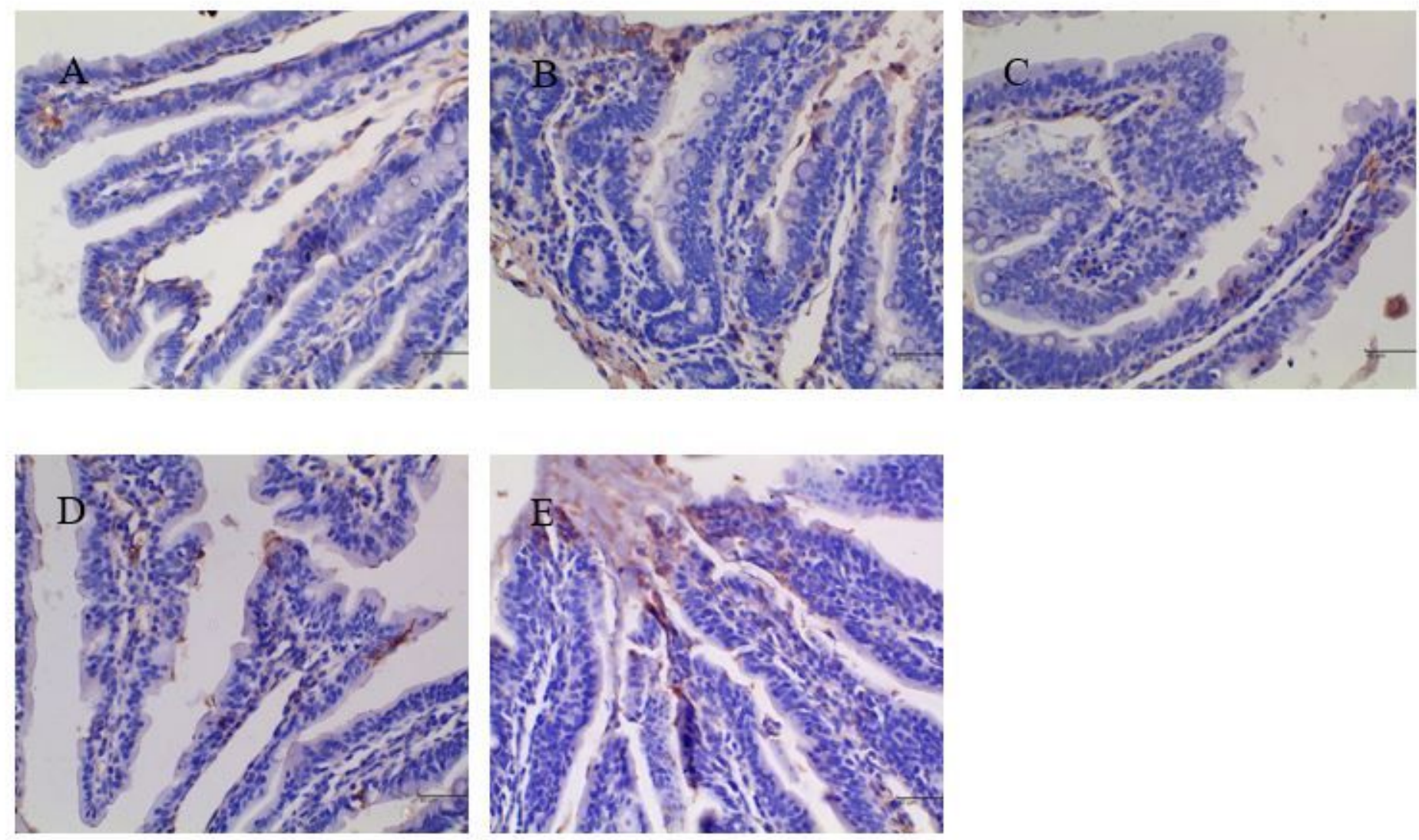

\section{Figure 6}

Immunohistochemical detection of SIgA of jejunum intestinal mucosa different groups $(0 \sim 30 \mathrm{~d})(\times 400)$. 

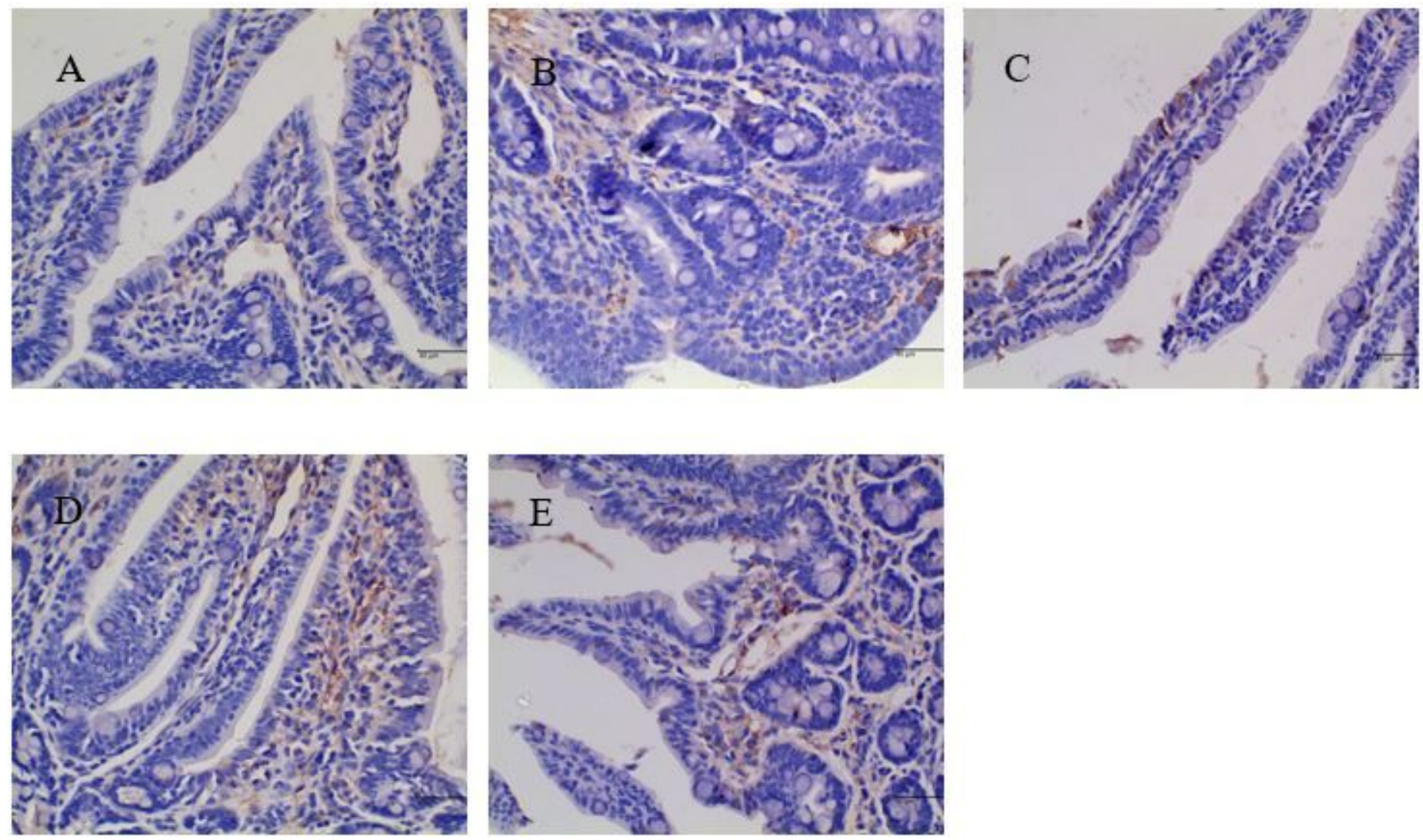

\section{Figure 7}

Immunohistochemical detection of SIgA of ileum intestinal mucosa different groups $(0 \sim 30 \mathrm{~d})(\times 400)$. 


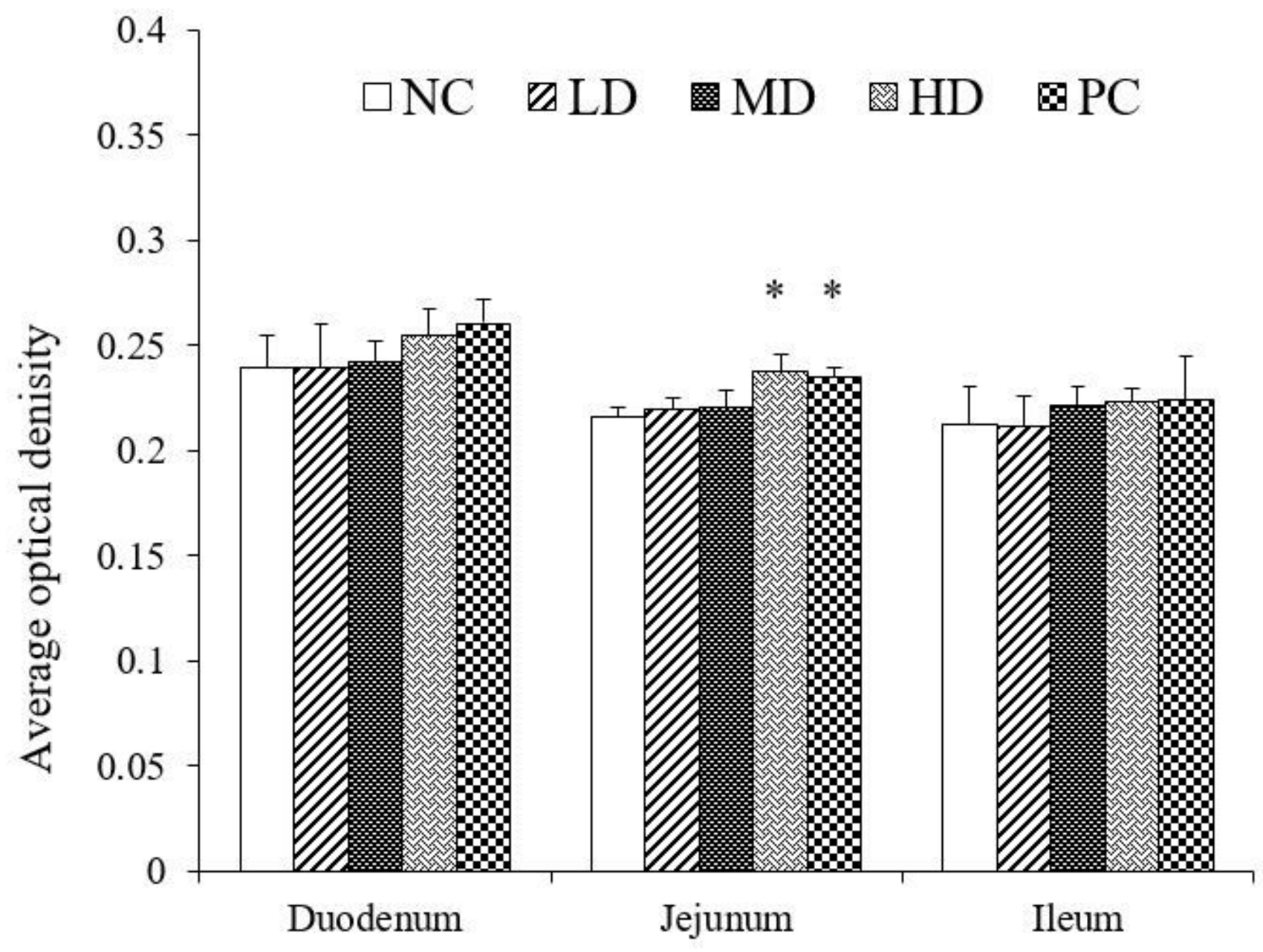

Figure 8

Average optical density of the express of SlgA in different groups on small intestinal mucosa $(n=6) .{ }^{*} p<$ 0.05 compared with NC. NC,control group; LD,0.5\% M-SJZG group; MD, 1.0\%M-SJZG group; HD, 2.0\% MSJZG group; PC, positive, $0.8 \%$ Gln group. 


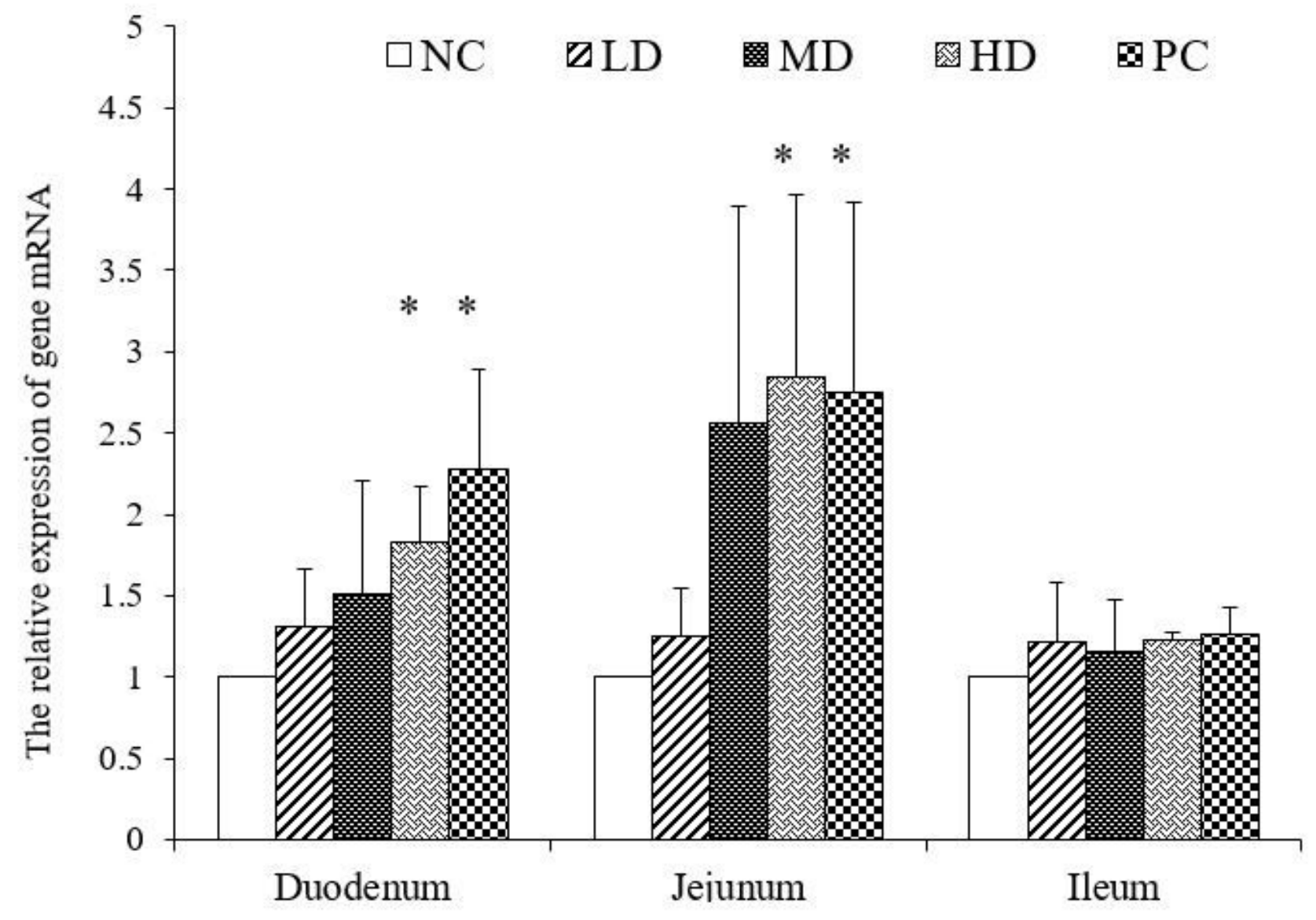

Figure 9

Effect of M-SJZG on gene expression of Zo1 in small intestinal mucosa of Rex Rabbits $(n=6) * p<0.05$ compared with NC. 


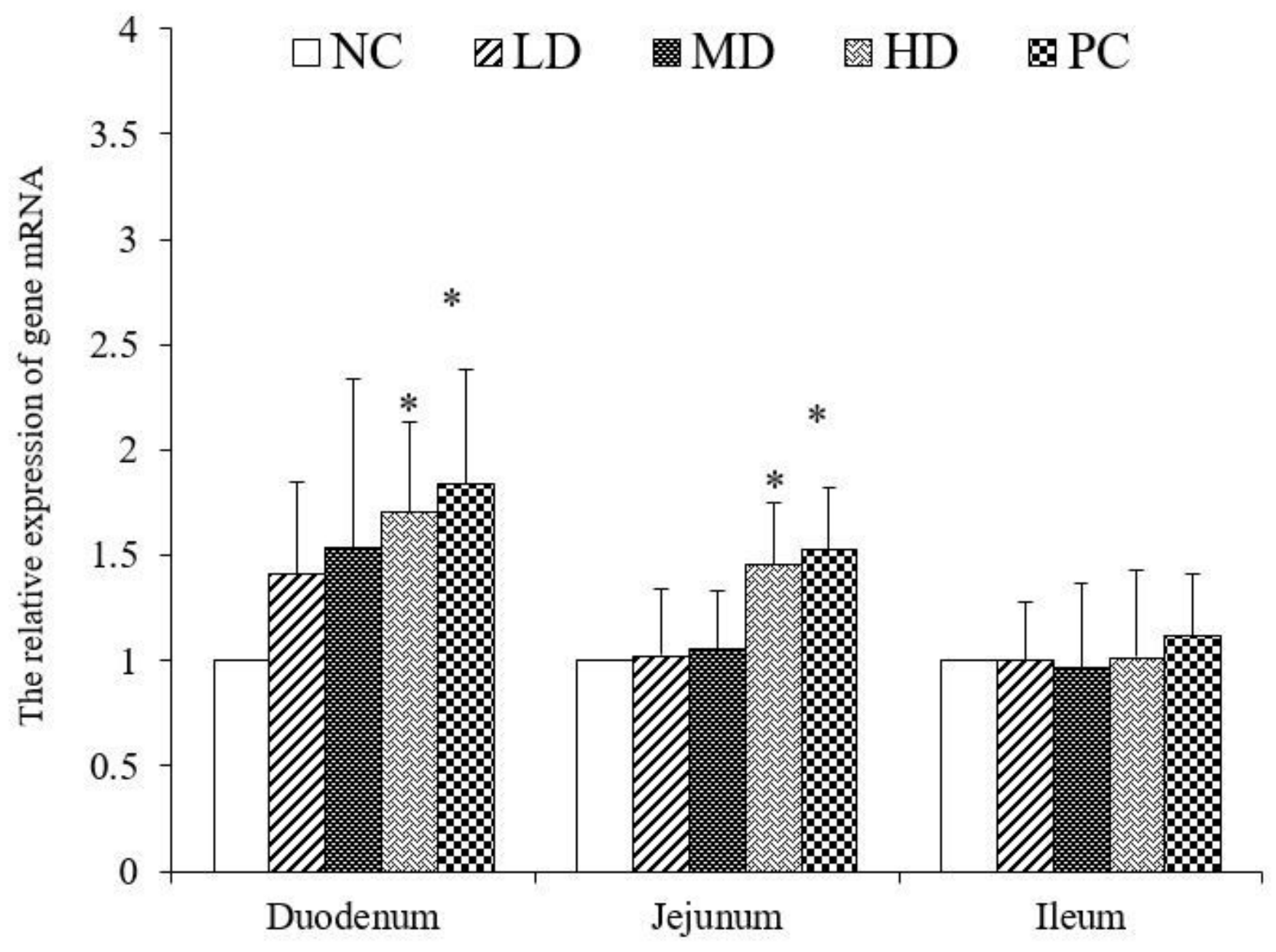

Figure 10

Effect of M-SJZG on gene expression of Claudin1 in small intestinal mucosa of Rex Rabbits $(n=6){ }^{*} p<$ 0.05 compared with NC. 


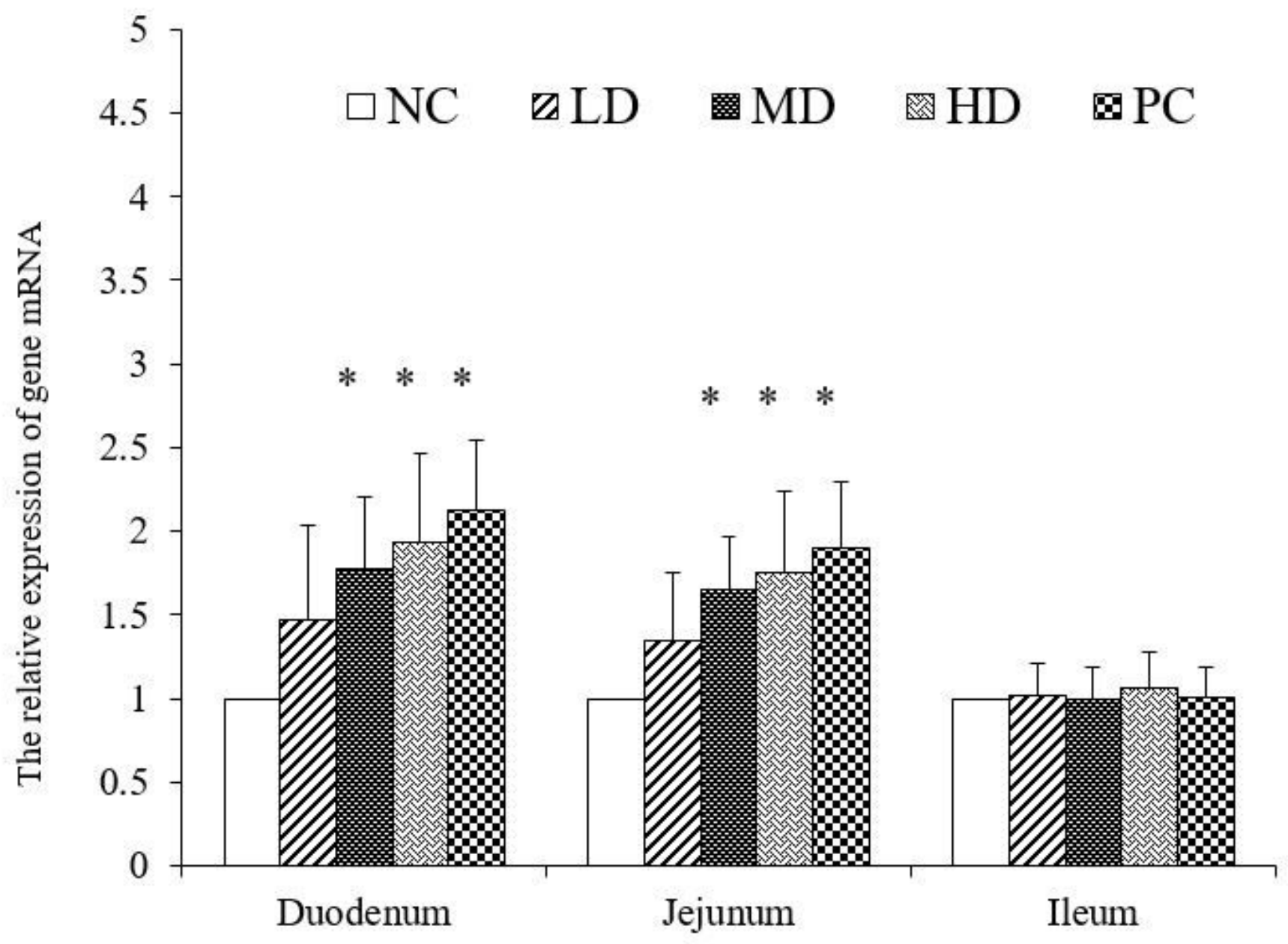

Figure 11

Effect of M-SJZG on gene expression of Occludin in small intestinal mucosa of Rex Rabbits $(n=6) * p<$ 0.05 compared with NC. 


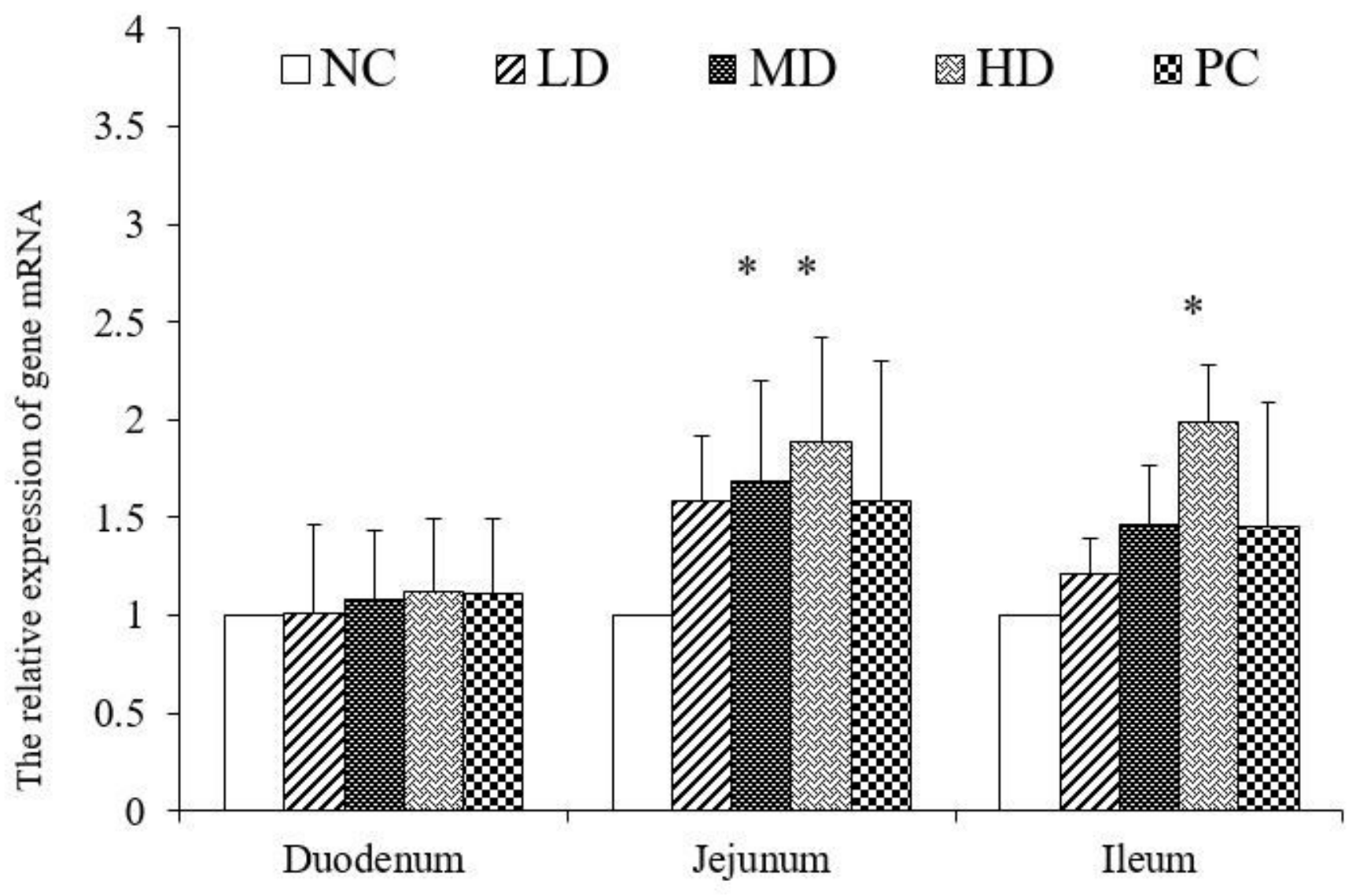

Figure 12

Effect of M-SJZG on gene expression of GLUT2 in small intestinal mucosa of Rex Rabbits $(n=6) * p<0.05$ compared with NC. 


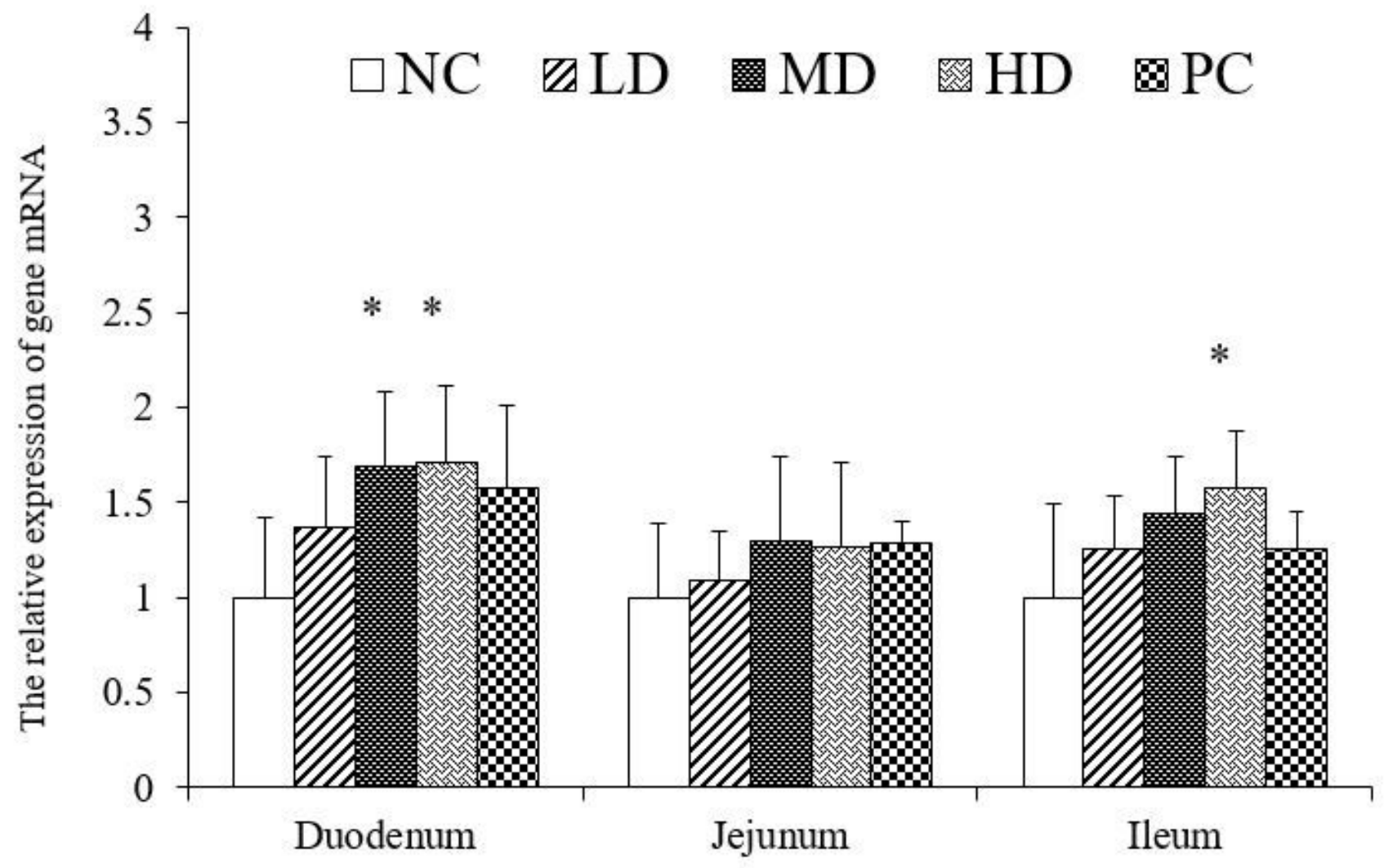

Figure 13

Effect of M-SJZG on gene expression of SGLT1 in small intestinal mucosa of Rex Rabbits $(n=6) * p<0.05$ compared with NC.

\section{Supplementary Files}

This is a list of supplementary files associated with this preprint. Click to download.

- graphicalabstract.tif

- graphicalabstract.tif

- supplementarymaterial.pdf

- supplementarymaterial.pdf 